\title{
Spontaneous rupture of an unscarred non-labouring gravid uterus at 32 weeks gestation
}

\author{
G R C Silva ${ }^{1}$, P Gange ${ }^{1}$, N Karunarathne ${ }^{1}$, N Senevirathne ${ }^{1}$ \\ Sri Lanka Journal of Obstetrics and Gynaecology 2012; 34: 53-54
}

\section{Introduction}

Ruptured uterus is an obstetric and surgical emergency that can lead to maternal or fetal death. Overall uterine rupture rate in pregnancy is $0.07 \%$ while rate of unscarred uterus is $0.012 \%$. The incidence is significantly higher in developing countries than developed countries. The maternal and fetal prognoses are bad especially when the rupture occurs in an unscarred uterus. Uterine rupture happens usually during delivery. Spontaneous rupture of an unscarred uterus is very rare. Uterine abnormalities, grandmultiparity, macrosomic fetus, cephalopelvic disproportion, malpresentation, neglected labour, trauma to the uterus from prior instrumentation such as in abortion, version and oxytocin stimulation are predisposing factors for uterine rupture. The commonest cause of rupture is dehiscence from previous section scar, especially in the presence of oxytocic stimulation. We hereby report a case of spontaneous uterine rupture which occurred at 32 weeks gestation in a non-labouring uterus with no previous risk factors.

\section{Case report}

A 29-year old mother in her 2nd pregnancy with one living child was admitted to our antenatal ward at De Zoyza Hospital for Women at 32 weeks gestation with non specific lower abdominal pain and loose stools for 2 days duration. Previous delivery was a vaginal delivery at 36 weeks of gestation following prelabour preterm rupture of membrane (birth weight $1.9 \mathrm{~kg}$ ). On examination abdomen was soft and mother was haemodynamically stable (temperature $98.4^{\circ} \mathrm{F}$, pulse 70 beats/minutes and regular, blood pressure 100/60). Haematological investigations were normal (haemoglobin $11.0 \mathrm{~g} / \mathrm{dl}$, white cell count 10700 /1, platelet count 276,000/1). Urine microscopy and stool full report were unremarkable. Fetal movements were satisfactory and CTG was normal. She was discharged from the ward after 2 days of admission as she was completely clinically asymptomatic.

\footnotetext{
${ }^{1}$ De Zoysa Hospital for Women, Colombo, Sri Lanka. Correspondence: GR C Silva

E-mail:grcruwan@gmail.com
}

She was readmitted on next day with insidious onset of severe lower abdominal pain for 8 hours, sudden onset vaginal bleeding and faintness. Her dating scan was normal. There was no history of trauma, any diagnostic or therapeutic intrauterine intervention. She had no significant past medical, surgical or gynaecological history. On examination, she was pale with respiratory rate of 28 per minute, pulse rate of 118 beats/minute and blood pressure of 90/60 mmHg. Abdomen was distended, tender and fetal parts were palpable prominently.

Speculum examination showed vaginal bleeding. There was marked tenderness on internal examination and cervix was uneffaced and os admitted tip of finger.

Ultrasound scan revealed fetal demise. An intact amniotic sac with normal volume of amniotic fluid was not clearly seen. With a provisional diagnosis of placental abruption or ruptured uterus, she was prepared for laparotomy. Blood investigations revealed haemoglobin of $8 \mathrm{gm} \%$ with no evidence of coagulopathy.

Emergency exploratory laparotomy was done with consent for hysterectomy or uterine repair under intravenous antibiotic cover. Amniotic sac was ruptured and dead male fetus (weight 2.2 kilograms) was found in the abdominal cavity. 1.0 liter of haemoperitoneum was found. Placenta was lying outside the uterus and appeared complete and normal. Uterus was contracted and there was a $12 \mathrm{~cm}$ irregular tear on the left side of the anterior aspect of the uterus extending from the lower segment to the fundus, longitudinally (Figure 1). There was no evidence of couvelaire uterus and no demonstrable congenital uterine anomaly. Rest of the pelvis looked normal, with no evidence of endometriosis or adhesions. The tear was repaired by suturing the uterus in double layer with vicryl. She received one unit of blood during intraoperative and two units during the post operative period.

As patient developed features of septicaemia on post operatively day 2 , she had undergone subtotal hysterectomy and specimen was sent for histopathologic examination. She was discharged from the hospital 7 days after surgery. 


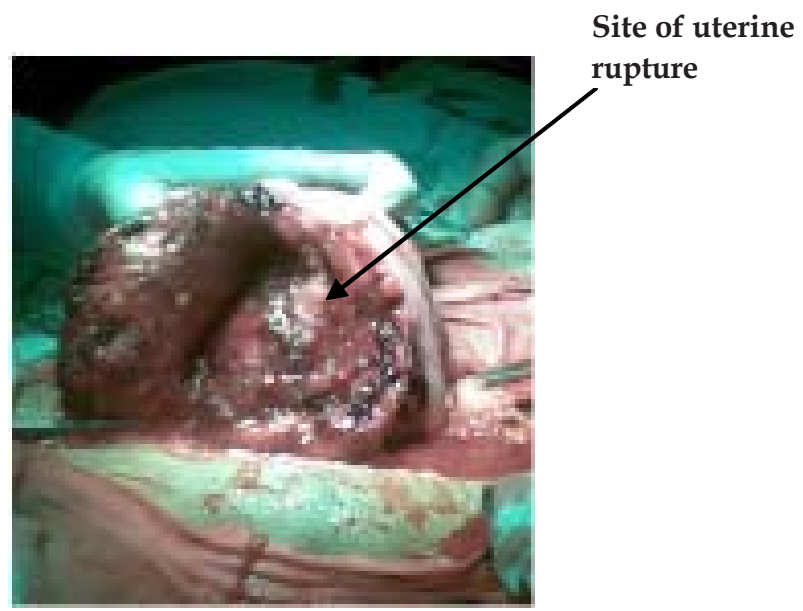

Figure 1

\section{Discussion}

Rupture of the non-labouring uterus is rare and is a potentially catastrophic event. Spontaneous ruptures are virtually always intrapartum and associated with risk factors such as multiparity, advanced maternal age, macrosomic fetus, cephalopelvic disproportion, malpresentation, scarred uterus, abnormal placentation such as placenta accreta, uterine abnormalities, uterine trauma from prior instrumentation from abortion, external cephalic or internal podalic version, oxytocin stimulation and instrumental deliveries. Risk factors associated with early uterine rupture include previous salpingectomy and cornual resection following ectopic pregnancy, trauma, myomectomy, congenital abnormalities and sacculation of the entrapped retroverted uterus. Uterine rupture can also occur in the absence of any of above these factors. A metaanalysis of seven modern studies revealed that in developed countries the rate of unscarred uterine rupture during pregnancy is $0.013 \%$ and an eightfoldincreased incidence $(0.11 \%)$ in developing countries ${ }^{2}$.

Patient denied any history of previous gynaecological surgery such as dilatation and curettage, termination of pregnancy or use of an intrauterine contraceptive device which could have resulted in undiagnosed uterine perforation, and a review of her previous case notes was unhelpful. To the best of our knowledge, this is one of the few cases documented rupture of a gravid uterus occurring before onset of labour without previously described risk factors. It has been suggested that predisposing risk factors to such unexpected uterine rupture may include uterine diverticulae, arteriovenous malformation and endometriosis. Histopathologic examination of uterus is not suggestive of diverticular disease of the uterus; moreover, diverticulosis of the bowel does not necessarily equate to uterine diverticulae, which is exceedingly rare. Arteriography is an important tool in the identification of arteriovenous malformation, but this was not carried out as we could not conserve the uterus in this case. Symptoms suggestive of the arteriovenous malformation, such as abnormal menstrual pattern or uterine hypotonia following delivery were absent in this case. There was no evidence to suggest that endometriosis was to be blamed for this rupture, since endometriotic changes were not seen at surgery. In the absence of any of the above risk factors, it thus makes us speculate that this rupture could have been due to some inherent weakening in the uterine myometrium, which became more profound in second pregnancy.

Initial signs and symptoms of uterine rupture are typically nonspecific, which makes diagnosis difficult and sometimes delays definitive therapy ${ }^{2}$.

Uterine rupture of the gravid uterus is associated with high maternal and fetal mortality and morbidity. Although it has been recommended that hysterectomy should be carried out in the ruptured gravid uterus to save the mother, we attempted to conserve the uterus at first laporotomy considering her future fertility wishes. Large longitudinal defect of the uterus might have lead to necrosis of the uterus causing septecaemia and hysterectomy was carried out at 2 nd laporotomy to save the mother's life.

\section{Conclusion}

This case re-emphasizes that uterine rupture can present in many different ways and high suspicion is required for timely intervention to prevent maternal and fetal, morbidity and mortality.

\section{References}

1. Gardeil F, Daly S, Turner MJ. Uterine rupture in pregnancy reviewed. Eur J Obstet Gynecol Reprod Biol 1994; 56(2): 107-10.

2. Nahum GG, Pham KQ. Uterine rupture in pregnancy. E Medicine from Webmed 2008, http://emedicine. medscape.com/article/275854-overview (accessed on April 14, 2011).

3. Dubuisson JB, Chavet X, Chapron C, et al. Uterine rupture after laparoscopic myomectomy. Hum Reprod 1995; 10: 1475-7.

4. Nkwabong E, Kouam L, Takang W. Spontaneous uterine rupture during pregnancy: case report and review of literature. Afr J Reprod Health 2007; 11(2): 107-10.

5. M'Lellan AN. Rupture of a pregnant diverticulum of the uterus. Glasgow Med J 1916; 3: 390-5.

6. Parry E, Wells M, Baxter T, Lane G. Recurrent spontaneous rupture in a nulliparous young woman. Br J Obstet Gynaecol 1995; 102: 420-1. 